\title{
On Models with Inverse-Square Exchange
}

\author{
Z. N. C. Ha and F. D. M. Haldane \\ Department of Physics, Princeton University, Princeton, New Jersey 08544
}

(Received 25 April, 1992)

\begin{abstract}
A one-dimensional quantum N-body system of either fermions or bosons with $S U(n)$ "spins" (or colors in particle physics language) interacting via inverse-square exchange is presented in this article. A class of eigenstates of both the continuum and lattice version of the model Hamiltonians is constructed in terms of the Jastrow-product type wave function. The class of states we construct in this paper corresponds to the ground state and the low energy excitations of the model that can be described by the effective harmonic fluid Hamiltonian. By expanding the energy about the ground state we find the harmonic fluid parameters (i.e. the charge, spin velocities, etc.), explicitly. The correlation exponent and the compressibility are also found. As expected the general harmonic relation(i.e. $\left.v_{S}=\left(v_{N} v_{J}\right)^{1 / 2}\right)$ is satisfied among the charge and spin velocities.
\end{abstract}

PACS numbers: 71.30.+h, 05.30.-d, 74.65.+n, 75.10.Jm

Typeset Using REVTEX 


\section{INTRODUCTION}

In this paper we report an exact treatment of a one-dimensional system of particles with SU(n) "spins" interacting with the inverse-square, two-body exchange in continuum space. In particular, we show that a class of eigenstates of the Hamiltonian is given by the Jastrow-product type of wave function. We also show that this model can be put on the lattice for all the positive integer values of the dimensionless interaction parameter which is defined in Eq. (2.1). The continuum version is a direct generalization of Sutherland's model of interacting spinless fermions (or hard core bosons) [1]. The lattice version corresponds to the $S U(n)$ t-J model which, at $n=1$, maps to the Haldane-Shastry model [2].

Fig. 1 shows how various models with the inverse square exchange have evolved from the original Sutherland's Model. In the figure the models that already exist are in rectangular boxes and the new models that we present in this paper are in ovals. The solid arrows mean "generalized from" or "evolved from". Two equivalent models that are represented in different ways are connected by a dashed arrow. We emphasize that while the bosonic t- $J$ model $(\lambda$ odd $)$ is a direct generalization of the bosonic supersymmetric case $(\lambda=1)$,

the fermionic t-J model ( $\lambda$ even) is not directly related to the fermionic supersymmetric t-J model that Kuramoto and Yokoyama [5] found.

\section{MULTICOMPONENT GENERALIZATION OF SUTHERLAND'S MODEL}

For convenience we consider a periodic system, where the Hamiltonian in units of $\hbar^{2} / m$ is given by

$$
H=-\frac{1}{2} \sum_{i} \frac{\partial^{2}}{\partial x_{i}^{2}}+\lambda \sum_{i<j} \frac{\lambda+P_{i j}^{\sigma}}{d\left(x_{i}-x_{j}\right)^{2}},
$$

where $\lambda$ is the dimensionless interaction parameter. $P_{i j}^{\sigma}$ is an operator that exchanges particle spins at $x_{i}$ and $x_{j}$, and $d(x)=(L / \pi)|\sin (\pi x / L)| \cdot d\left(x_{i}-x_{j}\right)$ is the chord distance between particles at $x_{i}$ and $x_{j}$ on a circle with circumference $L$. If all particles have the same spin, this model reduces to the system of spinless particles studied by Sutherland. Note that 
Sutherland's coupling parameter $\lambda^{\prime}$ corresponds to $\lambda-1$ in our notation. For example, the spinless free Fermi gas corresponds to $\lambda^{\prime}=1$, but in our notation $\lambda=0$.

It is easily found that the wave function $\Psi$ must vanish as $\left|x_{i}-x_{j}\right|^{\lambda+1}\left(\left|x_{i}-x_{j}\right|^{\lambda}\right)$ as $x_{i}-x_{j} \rightarrow 0$ in the case of symmetric (antisymmetric) spin configuration of the two particles at $x_{i}$ and $x_{j}$. And, if $0<\lambda<1$ the effective interaction strength is attractive for the antisymmetric spin configuration and there is some ambiguity in this case as it must be further specified whether $\Psi$ vanishes as $\left|x_{i}-x_{j}\right|^{\lambda}$ or as $\left|x_{i}-x_{j}\right|^{1-\lambda}$ as particles approach. If we choose the first boundary condition the free fermion limit is obtained as $\lambda \rightarrow 0$.

There are two possible interpretations for this system. The first is to consider the system as a ring embedded in a plane. Hence, even though the particles are constrained to move in one-dimension, the interaction is two-dimensional in nature. The other is to regard the system as strictly one-dimensional by taking $1 / d\left(x_{i}-x_{j}\right)^{2}$ as the effective interaction after summing the pairwise interaction of the particles around the ring infinite times. The following identity,

$$
\sum_{n=-\infty}^{+\infty} \frac{1}{(x+n L)^{2}}=\frac{1}{d(x)^{2}},
$$

has first been used by Sutherland [1] to show the validity of the latter interpretation.

In analogy with the states previously constructed for the $S U(2)$ spin chain in ref. [2], we propose the following Jastrow-product type wave functions for our Hamiltonian:

$$
\Psi(\{z \sigma\})=\Psi_{0} \prod_{k} z_{k}^{J_{\sigma_{k}}},
$$

where

$$
\begin{aligned}
\Psi_{0} & =\prod_{n>m} \phi_{n m} \\
\phi_{n m} & =\left|z_{n}-z_{m}\right|^{\lambda-x}\left(z_{n}-z_{m}\right)^{x+\delta_{\sigma_{n} \sigma_{m}}} \exp \left(i \frac{1}{2} \pi \operatorname{sgn}\left(\sigma_{n}-\sigma_{m}\right)\right) .
\end{aligned}
$$

Here, $\delta$ is the Kronecker delta function and $z_{n}=\exp \left(2 \pi i x_{n} / L\right) . \sigma_{n}$ is the ordered spin index and $J_{\sigma}$ the global current of particles with $\sigma$ spin. We take $J_{\sigma}$ to be an integer and will discuss the restrictions on the allowed values of $J_{\sigma}$ later in this article. Note also that the 
wave function with $\lambda=x=0$ is the Slater determinant that corresponds to the states of free $S U(n)$ fermions.

The symmetry of the wave function with respect to the exchange of particles is given as

$$
\Psi\left(\ldots, z_{i} \sigma_{i}, \ldots, z_{j} \sigma_{j}, \ldots\right)=(-1)^{x+1} \Psi\left(\ldots, z_{j} \sigma_{j}, \ldots, z_{i} \sigma_{i}, \ldots\right)
$$

Hence, for bosons(fermions), $x=1(x=0)$.

We write the total Hamiltonian as $H=H^{0}+H^{1}+H^{2}$ where $H^{0}, H^{1}$ and $H^{2}$ are the kinetic, potential and spin exchange Hamiltonian, respectively. We will show that each operator acting on the wave function gives two types of terms, "wanted" and "unwanted". "Wanted" terms are defined to be the terms that depend only on the global variables such as the total number of particles, $J_{\sigma}$, etc. "Unwanted" terms explicitly depend on the local variables such as $z_{i}$. Since the eigenenergy should depend only on the global variables, the "unwanted" terms for $H^{0}, H^{1}$ and $H^{2}$ should cancel or combine to give "wanted" terms.

We first examine $H^{0}$ acting on the wave function. We define the following derivatives:

$$
\begin{aligned}
\varphi_{i j} & \equiv \frac{\partial_{z_{j}} \phi_{i j}}{\phi_{i j}}=-\frac{\lambda}{z_{i}-z_{j}}-\frac{\lambda-x}{2 z_{j}}-\frac{\delta_{\sigma_{i} \sigma_{j}}}{z_{i}-z_{j}} \\
\xi_{i j} & \equiv \partial_{z_{j}} \varphi_{i j} \\
\eta_{j}^{(1)} & \equiv z_{j} \frac{\partial_{z_{j}} \Psi_{0}}{\Psi_{0}}=z_{j} \sum_{i(\neq j)} \varphi_{i j} \\
\eta_{j}^{(2)} & \equiv z_{j}^{2} \frac{\partial_{z_{j}}^{2} \Psi_{0}}{\Psi_{0}}=z_{j}^{2} \sum_{i(\neq j)} \xi_{i j}+\left(\eta_{j}^{(1)}\right)^{2} .
\end{aligned}
$$

In terms of these derivatives we find that $H^{0} \Psi=2(\pi / L)^{2} \sum_{j}\left[\eta_{j}^{(2)}+\left(2 J_{\sigma_{j}}+1\right) \eta_{j}^{(1)}+J_{\sigma_{j}}^{2}\right] \Psi$. After some algebra one can show that $H^{0}$ acting on the wave function gives "wanted" $\left(W_{K}\right)$ and "unwanted" $\left(U_{K}\right)$ terms. We write these terms as follow:

$$
H^{0} \Psi=2\left(\frac{\pi}{L}\right)^{2}\left(W_{K}+U_{K}\right) \Psi
$$

where

$$
W_{K}=\frac{\lambda^{2}}{12} N\left(N^{2}-1\right)+\frac{1}{2}(\lambda+1) \sum_{\sigma} M_{\sigma}\left(M_{\sigma}-1\right)+\frac{1}{3} \sum_{\sigma} M_{\sigma}\left(M_{\sigma}-1\right)\left(M_{\sigma}-2\right)
$$




$$
\begin{aligned}
& -\sum_{\sigma} M_{\sigma} J_{\sigma}\left(\lambda N-M_{\sigma}-J_{\sigma}-\lambda+1\right)+E(x), \\
U_{K} & =\lambda \sum_{i \neq j} \frac{z_{i} z_{j}}{\left(z_{i}-z_{j}\right)^{2}}\left\{\lambda-1+2 \delta_{\sigma_{i} \sigma_{j}}\right\}+\lambda \sum_{i \neq j} \frac{z_{i} J_{\sigma_{i}}-z_{j} J_{\sigma_{j}}}{z_{i}-z_{j}} \\
& +\lambda \sum_{(i \neq j \neq k)} \frac{z_{i}\left(z_{i}+z_{k}\right)}{\left(z_{i}-z_{j}\right)\left(z_{i}-z_{k}\right)} \delta_{\sigma_{i} \sigma_{j}},
\end{aligned}
$$

where $E(x)=x^{2} N(N-1)^{2} / 4+(x / 2)(N-1) \sum_{\sigma} M_{\sigma}\left(M_{\sigma}-1\right)+x(N-1) \sum_{\sigma} M_{\sigma} J_{\sigma} . M_{\sigma}$ is the total number of $\sigma$ particles in the system. We can now anticipate $U_{K}$ to be canceled or combined with the "unwanted" terms from $H^{1}$ and $H^{2}$ to give local variable independent terms.

$H^{1}$ acts trivially on $\Psi$ to give $\lambda^{2} \sum_{i<j} 1 / d\left(x_{i}-x_{j}\right)^{2}$. The action of $H^{2}$ on the wave function is less trivial. We need to evaluate the following expression:

$$
P_{\{M\}} \equiv \frac{1}{2 \Psi} \sum_{i>j} \frac{P_{i j}^{\sigma} \Psi}{d\left(x_{i}-x_{j}\right)^{2}}=-\frac{1}{\Psi(\{z \sigma\})} \sum_{i \neq j} \frac{z_{i} z_{j}}{\left(z_{i}-z_{j}\right)^{2}} \Psi\left(\left\{z \sigma^{\prime}\right\}\right),
$$

where $\left\{z \sigma^{\prime}\right\}$ is a configuration with $\sigma_{i}$ and $\sigma_{j}$ exchanged with respect to $\{z \sigma\}$. Similarly, we let $\left\{z^{\prime} \sigma\right\}$ be equal to $\{z \sigma\}$ with $z_{i}$ and $z_{j}$ exchanged. Using the identity, $\Psi(\{z \sigma\})=$ $(-1)^{x+1} \Psi\left(\left\{z^{\prime} \sigma^{\prime}\right\}\right)$, we may then rewrite the exchange operation as

$$
\begin{aligned}
P_{\{M\}} & =(-1)^{x+1} \sum_{i \neq j} \frac{z_{i} z_{j}}{\left(z_{i}-z_{j}\right)^{2}} \frac{\Psi\left(\left\{z \sigma^{\prime}\right\}\right)}{\Psi\left(\left\{z^{\prime} \sigma^{\prime}\right\}\right)} \\
& =\sum_{i \neq j} \frac{z_{i} z_{j}}{\left(z_{i}-z_{j}\right)^{2}}(-1)^{\delta_{\sigma_{i} \sigma_{j}}}\left(\frac{z_{i}}{z_{j}}\right)^{J_{\sigma_{j}}-J_{\sigma_{i}}} \prod_{k \neq i j}\left(\frac{z_{k}-z_{i}}{z_{k}-z_{j}}\right)^{\delta_{\sigma_{j} \sigma_{k}}-\delta_{\sigma_{i} \sigma_{k}}}
\end{aligned}
$$

The action of $P^{\sigma}$ is same for both boson and fermion cases and is independent of the interaction parameter $\lambda$.

We use the following identity,

$$
\left(\frac{z}{z^{\prime}}\right)^{n} \equiv \sum_{q=1}^{|n|}\left(\begin{array}{c}
|n| \\
q
\end{array}\right)\left\{\left(\frac{z-z^{\prime}}{z^{\prime}}\right)^{q} \theta(n)+\left(\frac{z^{\prime}-z}{z}\right)^{q} \theta(-n)\right\},
$$

to rewrite Eq. (2.14) as

$$
P_{\{M\}}=P_{0}+\sum_{\sigma, \sigma^{\prime}} \sum_{q=1}^{\left|J_{\sigma^{\prime}}-J_{\sigma}\right|} P_{q}^{\sigma \sigma^{\prime}}
$$

$\theta$ in Eq. 2.15) is the step function with $\theta(0)=1 / 2$, and $P_{0}$ and $P_{q}^{\sigma \sigma^{\prime}}$ are given by 


$$
\begin{aligned}
P_{0} & =-\sum_{i \neq j} \frac{z_{i} z_{j}}{\left(z_{i}-z_{j}\right)^{2}}\left\{\delta_{\sigma_{i} \sigma_{j}}+\left(1-\delta_{\sigma_{i} \sigma_{j}}\right) \prod_{k \neq i j}\left(\frac{z_{k}-z_{i}}{z_{k}-z_{j}}\right)^{\delta_{\sigma_{j} \sigma_{k}}-\delta_{\sigma_{i} \sigma_{k}}}\right\} \\
P_{q}^{\sigma \sigma^{\prime}} & =2 \sum_{i \neq j} \frac{z_{i} z_{j}}{\left(z_{i}-z_{j}\right)^{2}} \delta_{\sigma \sigma_{i}} \delta_{\sigma^{\prime} \sigma_{j}}\left(1-\delta_{\sigma \sigma^{\prime}}\right) \prod_{k \neq i j}\left(\frac{z_{k}-z_{i}}{z_{k}-z_{j}}\right)^{\delta_{\sigma_{j} \sigma_{k}}-\delta_{\sigma_{i} \sigma_{k}}} \\
& \times\left(\begin{array}{c}
J_{\sigma^{\prime}}-J_{\sigma} \\
q
\end{array}\right) \theta\left(J_{\sigma^{\prime}}-J_{\sigma}\right)\left(\frac{z_{i}-z_{j}}{z_{j}}\right)^{q} .
\end{aligned}
$$

It is useful to separately consider the terms with $q=0,1$ and the terms with $q \geq 2$. For the terms with $q=0,1$ we introduce two sets of site indices $\{\alpha\}$ and $\{\beta\}$. The set $\{\alpha\}(\{\beta\})$ includes all the locations of particles with the spin $\sigma\left(\sigma^{\prime}\right)$. Using the identity,

$$
\left(\frac{z_{k}-z_{i}}{z_{k}-z_{j}}\right)^{\delta_{\sigma^{\prime} \sigma_{k}}-\delta_{\sigma \sigma_{k}}} \equiv 1-\delta_{\sigma^{\prime} \sigma_{k}} \frac{z_{i}-z_{j}}{z_{k}-z_{i}}+\delta_{\sigma \sigma_{k}} \frac{z_{i}-z_{j}}{z_{k}-z_{j}} \text { for } \sigma \neq \sigma^{\prime}
$$

the products in $P_{\{M\}}$ may be expanded and the typical terms in $P_{\{M\}}$ can be simplified using the following two theorems.

Theorem 1: Let $\{\alpha\}$ and $\{\beta\}$ be two sets of distinct integers between 1 and $N$, and let $0 \leq q \leq 1$ and $\Delta=\left(1-\delta_{\sigma, \sigma^{\prime}}\right) \delta_{\sigma \sigma_{i}} \delta_{\sigma \sigma_{\alpha}} \cdots \delta_{\sigma \sigma_{\alpha_{n}}} \delta_{\sigma^{\prime} \sigma_{j}} \delta_{\sigma^{\prime} \sigma_{\beta}} \cdots \delta_{\sigma^{\prime} \sigma_{\beta m}}$. Then,

$$
\begin{aligned}
\sum_{n=1}^{M_{\sigma}-1} & \sum_{m=1-q}^{M_{\sigma^{\prime}}-1} \sum_{i \neq j} \sum_{\{\alpha\},\{\beta\}} \frac{(-1)^{m}}{n ! m !} \frac{z_{i} z_{j}^{1-q}\left(z_{i}-z_{j}\right)^{n+m-2+q}}{\left(z_{\alpha_{1}}-z_{i}\right) \cdots\left(z_{\alpha_{n}}-z_{i}\right)\left(z_{\beta_{1}}-z_{j}\right) \cdots\left(z_{\beta_{m}}-z_{j}\right)} \Delta \\
= & \left\{\begin{array}{l}
-\sum_{k=1}^{\operatorname{Min}\left(M_{\sigma}, M_{\sigma^{\prime}}\right)}\left(M_{\sigma}-k\right)\left(M_{\sigma^{\prime}}-k\right) \text { for } q=0, \\
-\sum_{k=1}^{\operatorname{Min}\left(M_{\sigma}, M_{\sigma^{\prime}}\right)}\left(M_{\sigma}-k\right) \text { for } q=1 .
\end{array}\right.
\end{aligned}
$$

Theorem 2: For $q \geq 2$ the following identity will hold for $M_{\sigma} \geq M_{\sigma^{\prime}}$,

$$
\begin{aligned}
\sum_{i \neq j} & \frac{z_{i}}{z_{j}}\left(\frac{z_{i}-z_{j}}{z_{j}}\right)^{q-2}\left(1-\delta_{\sigma \sigma^{\prime}}\right) \delta_{\sigma \sigma_{i}} \delta_{\sigma^{\prime} \sigma_{j}} \prod_{k \neq i j}\left(\frac{z_{k}-z_{i}}{z_{k}-z_{j}}\right)^{\delta_{\sigma^{\prime} \sigma_{k}}-\delta_{\sigma \sigma_{k}}} \\
= & \left\{\begin{array}{l}
M_{\sigma^{\prime}} \text { for } q=2 \\
0 \text { for } 2<q \leq M_{\sigma}-M_{\sigma^{\prime}}+1 .
\end{array}\right.
\end{aligned}
$$

Proofs of these theorems are given in Appendix A and B.

After reorganizing some terms we obtain the following results:

$$
P_{\{M\}}=W_{P}+U_{P}
$$

where 


$$
\begin{aligned}
W_{P} & =-\frac{1}{3} \sum_{\sigma} M_{\sigma}\left(M_{\sigma}-1\right)\left(M_{\sigma}-2\right)-\frac{1}{3} \sum_{\sigma<\sigma^{\prime}} M_{\sigma^{\prime}}\left(M_{\sigma^{\prime}}-1\right)\left(3 M_{\sigma}-M_{\sigma^{\prime}}-1\right), \\
& -\sum_{\sigma<\sigma^{\prime}} M_{\sigma^{\prime}}\left(M_{\sigma}-M_{\sigma^{\prime}}\right)\left(J_{\sigma^{\prime}}-J_{\sigma}\right)+\sum_{\sigma<\sigma^{\prime}} M_{\sigma^{\prime}}\left(J_{\sigma^{\prime}}-J_{\sigma}\right)^{2} \\
U_{P} & =\sum_{i \neq j} \frac{z_{i} z_{j}}{\left(z_{i}-z_{j}\right)^{2}}\left\{1-2 \delta_{\sigma_{i} \sigma_{j}}\right\}+\sum_{i \neq j} \frac{z_{i} J_{\sigma_{j}}-z_{i} J_{\sigma_{i}}}{z_{i}-z_{j}} \\
& -2 \sum_{(i \neq j \neq k)} \frac{z_{i} z_{j}}{\left(z_{i}-z_{j}\right)\left(z_{i}-z_{k}\right)} \delta_{\sigma_{i} \sigma_{k}} .
\end{aligned}
$$

Because of symmetry we can choose $M_{1} \geq M_{2} \geq \ldots \geq M_{n}$ and $0 \leq J_{\sigma^{\prime}}-J_{\sigma} \leq M_{\sigma}-M_{\sigma^{\prime}}+1$ for $\sigma^{\prime}>\sigma$ without loss of generality. And, one can easily check that $U_{K}, U_{P}$ and the potential energy term combine to give local-variable independent terms.

Before we give the full expressions for the eigenenergy, we discuss the allowed values of the integer currents. Theorem 2 gives a simple rule for selecting allowed currents. If we choose $-1 \leq J_{\sigma^{\prime}}-J_{\sigma} \leq M_{\sigma}-M_{\sigma^{\prime}}+1$ for $M_{\sigma} \geq M_{\sigma^{\prime}}$, then $P_{q}=0$ for $q>2$. Otherwise, the energy will not in general be independent of the local variables and the corresponding wave function will not be an eigenfunction. Pictorial illustration of the allowed currents is shown in Fig. 3. Each row of $M_{\nu}$ boxes represents $M_{\nu}$ "particles" of same spin. A single box gives a unit of current. Fig. $3(\mathrm{a})$ is the reference state with no current. If the current is $j>0(<0)$ for a row, the row is moved $j$ boxes to the right(left) as shown in Fig. 3(b). In order for a state to be an eigenstate, the following condition must hold true: For a given pair of rows of boxes all except the first and the last boxes in the row with smaller number of boxes must be positioned within the other row. Fig. $3(\mathrm{c})$, for example, cannot be an eigenstate because the last two boxes in the second row are not within the first row.

Finally, the eigenenergies of the model can be given as follow:

$$
E=\frac{2 \hbar^{2} \pi^{2}}{m L^{2}}\left(E_{1}+E_{2}+E(x)\right)
$$

where $E_{1}$ and $E_{2}$ are energies due to one- and two-spin interactions, respectively, and are given by

$$
E_{1}=\frac{\lambda^{2}}{12} N\left(N^{2}-1\right)+\frac{1}{2} \lambda N \sum_{\sigma} M_{\sigma}\left(M_{\sigma}-1\right)+\frac{1}{6}(1-\lambda) \sum_{\sigma} M_{\sigma}\left(M_{\sigma}-1\right)\left(2 M_{\sigma}-1\right)
$$




$$
\begin{aligned}
& +\sum_{\sigma} J_{\sigma} M_{\sigma}\left(M_{\sigma}+J_{\sigma}-1\right) \\
E_{2} & =-\frac{1}{3} \lambda \sum_{\sigma<\sigma^{\prime}} M_{\sigma^{\prime}}\left(M_{\sigma^{\prime}}-1\right)\left(3 M_{\sigma}-M_{\sigma^{\prime}}-1\right)-\lambda \sum_{\sigma<\sigma^{\prime}} M_{\sigma^{\prime}}\left(M_{\sigma}-M_{\sigma^{\prime}}\right)\left(J_{\sigma^{\prime}}-J_{\sigma}\right) \\
& +\lambda \sum_{\sigma<\sigma^{\prime}} M_{\sigma^{\prime}}\left(J_{\sigma^{\prime}}-J_{\sigma}\right)^{2}
\end{aligned}
$$

The ground state for fermions(bosons) is given by the following two conditions: $(i) M_{\sigma^{\prime}}=$ $M_{\sigma}=M$ and $(i i) J_{\sigma^{\prime}}=J_{\sigma}=J=-(M-1) / 2, \quad-(N+M-1) / 2$ for fermions and bosons,

respectively. The currents should be integers. If the condition $(i i)$ does not give an integer current the ground state is degenerate and the ground state currents are two integers closest to the the half odd integer value.

\section{SU(N) T-J MODEL}

In ref. [2], Sutherland's model for a system of spinless particles in continuum space was extended to the lattice. Using the similar procedures we put our model on the lattice. We propose the following Hamiltonian:

$$
H=\mathcal{P}\left(\sum_{j=1}^{N} \sum_{n=1}^{N_{a}-1} \frac{t_{j}^{n}}{d(n)^{2}}+\sum_{i>j} \frac{l\left(l+P_{i j}^{\sigma}\right) n_{i} n_{j}}{d\left(x_{i}-x_{j}\right)^{2}}\right) \mathcal{P} .
$$

$l$ is a positive integer. $N_{a}$ is the total number of sites and $\mathrm{N}$ the total number of particles. $d(n)=\left(N_{a} / \pi\right)|\sin (n)| . t_{j}^{n}$ hops a particle at $j$ th site to $(j+n)$ th $\left(\bmod N_{a}\right)$ site. $n_{j}$ is equal to one(zero) if $j$ th site is occupied (empty). $P_{i j}^{\sigma}$ is, as before, the spin exchange operator. $\mathcal{P}$ is the projection operator that insures the absence of multiply occupied sites.

In analogy with ref. [2] we propose the following eigenstate for the model Hamiltonian,

$$
\left|\Psi_{\{M\}}\right\rangle=\sum_{\{z \sigma\}} \phi(\{z \sigma\})|\{z \sigma\}\rangle .
$$

Since the total number of each spin is a good quantum number we classify the eigenstates into sectors labeled by $\{M\} \equiv\left(M_{1}, M_{2}, \ldots, M_{n}\right)$, where $M_{\nu}$ is the number of

$\nu$ th spin and $\sum_{\nu=1}^{n} M_{\nu}=N$. We also represent the particle configuration as $\{z \sigma\} \equiv$ $\left(z_{1} \sigma_{1}, \ldots, z_{i} \sigma_{i}, \ldots, z_{j} \sigma_{j}, \ldots, z_{N} \sigma_{N}\right)$, where $z_{i}$ and $\sigma_{i}$ are the location and spin of the $i$ th 
particle. The sum in Eq. (3.2) is over $N_{a} ! /\left(M_{h} ! M_{1} ! \cdots M_{n} !\right)$ distinct spin configurations of the sector $\{M\}$ where $N_{a}$ and $M_{h}$ are the total number of sites and the number of holes, respectively. The function $\phi$ in Eq. (3.2) is given by the following Jastrow-product,

$$
\phi(\{z\} \mid\{\sigma\})=\prod_{i<j}\left(z_{i}-z_{j}\right)^{l+\delta_{\sigma_{i} \sigma_{j}}} e^{i \frac{\pi}{2} \operatorname{sgn}\left(\sigma_{i}-\sigma_{j}\right)} \prod_{k} z_{k}^{J_{\sigma_{k}}}
$$

Considering the symmetry of the wave function we take odd (even) $l$ to be bosonic (fermionic) state.

As in the continuum case we break up the total Hamiltonian into the kinetic, potential, and spin exchange parts $\left(H_{L}^{0}, H_{L}^{1}\right.$ and $H_{L}^{3}$, respectively). The actions of $H_{L}^{1}$ and $H_{L}^{3}$ are same as the corresponding operators in the continuum case. For $H_{L}^{0}$ we have the following relations:

$$
\begin{aligned}
<H^{0}> & \equiv \frac{\left\langle z_{1} \sigma_{1}, \ldots, z_{N} \sigma_{N}\left|H^{0}\right| \Psi_{\{M\}}\right\rangle}{\left\langle z_{1} \sigma_{1}, \ldots, z_{N} \sigma_{N} \mid \Psi_{\{M\}}\right\rangle} \\
& =4 \sum_{i=1}^{N} \sum_{n=1}^{N_{a}-1} z^{n J_{\sigma_{i}}}\left(1-z^{n}\right)^{-1}\left(1-z^{-n}\right)^{-1} \prod_{j(\neq i)}\left(\frac{z_{i} z^{n}-z_{j}}{z_{i}-z_{j}}\right)^{l+\delta_{\sigma_{i} \sigma_{j}}}
\end{aligned}
$$

where $\left|z_{1} \sigma_{1}, \ldots, z_{N} \sigma_{N}\right\rangle$ is one of the basis states of the sector $\left|\Psi_{\{M\}}\right\rangle$. In order to evaluate $<H_{L}^{0}>$ we need the following theorem (a slightly different version of this theorem can be found in ref. [2]).

Theorem 3: Let $J$ and $p$ be non-negative integers and $z=\exp \left(2 \pi i / N_{a}\right)$. Then,

$$
\begin{aligned}
\sum_{n=1}^{N_{a}-1} z^{n J}\left(1-z^{-n}\right)^{-1}\left(1-z^{n}\right)^{p-1} & =\frac{N_{a}^{2}-1}{12}-\frac{J\left(N_{a}-J\right)}{2} \text { for } p=0 \\
& =-J+\frac{N_{a}-1}{2} \text { for } p=1 \\
& =+1 \text { for } p=2,0 \leq J \leq N_{a}-2 \\
& =-\left(N_{a}-1\right) \text { for } p=2, J=N_{a}-1 \\
& =\sum_{m>0}(-1)^{m N_{a}-J}\left(\begin{array}{c}
p-2 \\
m N_{a}-J-1
\end{array}\right) N_{a} \text { for } p \geq 3
\end{aligned}
$$

$\mathrm{m}$ is a positive integer, and the restriction on the current is $0 \leq J \leq N_{a}-1$ unless specified otherwise. 
If we take $l>0$, we can multiply out the product in Eq. (3.4). The resulting expression will be a sum of terms containing a factor $\left(1-z^{n}\right)^{p}$. Since the maximum value of $p$ is $l(N-1)+M_{\sigma}-1$, if we choose $0 \leq J_{\sigma} \leq N_{a}-l(N-1)-M_{\sigma}+1$, we may ignore terms with $p \geq 3$. We combine the remaining terms with those from $H_{L}^{1}$ and $H_{L}^{2}$ and obtain the following eigenenergies for the lattice model:

$$
E^{L}=E_{1}^{L}+E_{2}^{L}
$$

where

$$
\begin{aligned}
E_{1}^{L} & =\frac{1}{6} N\left(N_{a}^{2}-1\right)+\frac{l^{2}}{3} N(N-1)(N-2)-\frac{l}{2}\left(N_{a}-l\right) N(N-1) \\
& -\left(\frac{N_{a}-1}{2}-l(N-1)\right) \sum_{\sigma} M_{\sigma}\left(M_{\sigma}-1\right)+\frac{1}{3}(1-l) \sum_{\sigma} M_{\sigma}\left(M_{\sigma}-1\right)\left(M_{\sigma}-2\right) \\
& -\sum_{\sigma} M_{\sigma} J_{\sigma}\left(N_{a}-l N-M_{\sigma}-J_{\sigma}+l+1\right), \\
E_{2}^{L} & =E_{2},
\end{aligned}
$$

where $E_{2}$ is given by Eq. (2.25). We emphasize at this point that the above expressions for the eigenenergies for the lattice model are valid only for $l N+M_{\sigma} \leq N_{a}+l+1$.

For $l=1$ (bosonic supersymmetric case) we can find another expression for the energy for $N+M_{\sigma} \geq N_{a}+2$. We proceed by rewriting the kinetic energy term as

$$
<H^{0}>=2 \sum_{\sigma} \sum_{i \neq j}\left(1-\delta_{\sigma \sigma_{h}}\right) \delta_{\sigma \sigma_{i}} \delta_{\sigma_{h} \sigma_{j}}\left(\frac{z_{i}}{z_{j}}\right)^{J_{\sigma}+1} \frac{z_{i} z_{j}}{\left(z_{i}-z_{j}\right)^{2}} \prod_{k \neq i j}\left(\frac{z_{k}-z_{i}}{z_{k}-z_{j}}\right)^{\delta_{\sigma_{h} \sigma_{k}}-\delta_{\sigma \sigma_{k}}},
$$

where $\sigma_{h}$ represents the hole. The above expression is almost identical with the expression for the spin exchange operator and can easily be evaluated using Theorem 1 and 2. The energy then is given by

$$
E(l=1)=\frac{\pi^{2}}{2 N_{a}^{2}}\left(E_{k}(l=1)+W_{P}\right)
$$

where

$$
\begin{aligned}
E_{k}(l & =1)=-\frac{M_{h}\left(N_{a}^{2}-1\right)}{6}-\frac{1}{3} M_{h}\left(M_{h}-1\right)\left(M_{h}-2\right)+M_{h} \sum_{\sigma} J_{\sigma}\left(J_{\sigma}-M_{\sigma}-1\right) \\
& +\frac{N_{a}-1}{2}\left(\sum_{\sigma} M_{\sigma}\left(M_{\sigma}-1\right)+M_{h}\left(M_{h}-1\right)\right)-\frac{1}{3} M_{h}\left(M_{h}-1\right)\left[3 N-n\left(M_{h}+1\right)\right] \\
& +M_{h} N+\sum_{\sigma}\left(J_{\sigma}-1\right)\left[2 M_{\sigma} M_{h}-M_{h}\left(M_{h}+1\right)\right],
\end{aligned}
$$


where the currents are restricted to $-\left(M_{\sigma}-M_{h}\right) \leq J_{\sigma} \leq 0 . W_{P}$ in Eq. (3.9) is given by Eq. (2.21).

For $l=0$ the wave function in Eq. (3.2) is that of Gutzwiller projected free fermions and no longer an eigenstate of the Hamiltonian in Eq. (3.1). However, the wave function is an eigenstate of the following Hamiltonian

$$
H=\mathcal{P}\left(-\sum_{j, n} \frac{t_{j}^{n}}{d(n)^{2}}+\sum_{i>j} \frac{\left(P_{i j}^{\sigma}-1\right) n_{i} n_{j}}{d\left(x_{i}-x_{j}\right)^{2}}\right) \mathcal{P}
$$

This Hamiltonian is $\mathrm{SU}(\mathrm{n})$ generalization of the $\mathrm{SU}(2)$ fermionic supersymmetric t-J model which Kuramoto and Yokoyama [5] solved. If we employ the similar technique used for $l=1$ case we may find the eigenenergy given by Eq. (3.9) with $E_{k}(l=1)$ replaced with $E_{k}(l=0)$ which is given by

$$
E_{k}(l=0)=-\frac{N\left(N_{a}^{2}-1\right)}{6}+\frac{1}{2}\left(N_{a}-1\right) \sum_{\sigma} M_{\sigma}\left(M_{\sigma}-1\right)+M_{h} \sum_{\sigma} J_{\sigma}\left(M_{\sigma}+J_{\sigma}-1\right) .
$$

The currents are integers restricted to $-M_{\sigma} \leq J_{\sigma} \leq 0$. The ground state energy is obtained if $J_{\sigma}=-\left(M_{\sigma}-1\right) / 2$ and $M_{\sigma}$ odd. If $M_{\sigma}$ are not all odd, the ground state is degenerate.

\section{SU(N) SPIN CHAIN}

If we take the spin exchange Hamiltonian as our full Hamiltonian for a system of spins on a lattice ring, we obtain the $S U(n)$ spin chain model. This is equivalent to taking the "half filling" limit $\left(N \rightarrow N_{a}\right)$ of the lattice t-J model. In this model the eigenenergies for the boson and fermion systems are identical. Since every site is occupied with a spin, $U_{P}$ in Eq. (2.22) can now be summed and is equal to $-N_{a}\left(N_{a}^{2}-1\right) / 12+\left\{\left(N_{a}-1\right) / 2\right\} \sum_{\sigma} M_{\sigma}\left(M_{\sigma}-1\right)$. Hence, $U_{P}$ is now local variable independent, and the eigenenergy is given by Eq. (2.20).

\section{THE HARMONIC FLUID DESCRIPTION}

We now turn our attention to the low energy excitation spectrum of our model in the thermodynamic limit. The low energy excitation spectrum of a one-dimensional quantum 
fluid may be described by a sum of two independent harmonic fluid Hamiltonians, one for the charge and the other for the spin. This is a slight generalization of the spinless particle system in ref. [3]. In general the effective Hamiltonian can be written as follow:

$$
H=\sum_{\sigma, \sigma^{\prime}} \int d x\left(A_{\sigma \sigma^{\prime}} \Pi_{\sigma}(x) \Pi_{\sigma^{\prime}}(x)+B_{\sigma \sigma^{\prime}} \nabla \phi_{\sigma}(x) \nabla \phi_{\sigma^{\prime}}(x)\right)
$$

$\Pi_{\sigma}(x)$ is the local density fluctuation field as in ref. [3]. $\phi_{\sigma}(x)$ is the canonical conjugate field to $\Pi(x),\left[\phi_{\sigma}(x), \Pi_{\sigma^{\prime}}\left(x^{\prime}\right)\right]=i \delta_{\sigma \sigma^{\prime}} \delta\left(x-x^{\prime}\right)$. Because of the $S U(n)$ symmetry we have $A_{\sigma \sigma^{\prime}}=a_{1}+a_{0} \delta_{\sigma \sigma^{\prime}}$ and $B_{\sigma \sigma^{\prime}}=b_{1}+b_{0} \delta_{\sigma \sigma^{\prime}}$. We now express the Hamiltonian in terms of the Fourier transformed fields, $\Pi_{\sigma k}$ and $\phi_{\sigma k}$.

$$
H=\sum_{k} \sum_{\sigma, \sigma^{\prime}} A_{\sigma \sigma^{\prime}} \Pi_{\sigma k} \Pi_{\sigma^{\prime}-k}+B_{\sigma, \sigma^{\prime}} k^{2} \phi_{\sigma k} \phi_{\sigma^{\prime}-k}
$$

We now construct normal mode fields as $\Pi_{k}^{\nu}=\sum_{\sigma} a_{\sigma}^{\nu} \Pi_{\sigma k}$ and $\phi_{k}^{\nu}=\sum_{\sigma} b_{\sigma}^{\nu} \phi_{\sigma k}$. If we choose $\sum_{\sigma} b_{\sigma}^{\nu} a_{\sigma}^{\nu^{\prime}}=\delta_{\nu \nu^{\prime}}$, then $\left[\phi_{k}^{\nu}, \Pi_{-k^{\prime}}^{\nu^{\prime}}\right]=i \delta_{k k^{\prime}} \delta_{\nu \nu^{\prime}}$. We have the following equations of motion: $\left[H,\left[H, \Pi_{k}^{\nu}\right]\right]=-\left(v^{\nu} k\right)^{2} \Pi_{k}^{\nu}, \quad\left[H,\left[H, \phi_{k}^{\nu}\right]\right]=-\left(v^{\nu} k\right)^{2} \phi_{k}^{\nu}$. For $\Pi$-field we obtain the following equation,

$$
\left(a_{0} b_{0}-\left(v^{\nu}\right)^{2}\right) a_{\sigma}^{\nu}+\left(a_{1} b_{0}+a_{0} b_{1}+n a_{1} b_{1}\right) \sum_{\beta} a_{\beta}^{\nu}=0
$$

The same equation with $a_{\sigma}^{\nu}$ replaced with $b_{\sigma}^{\nu}$ holds for $\phi$-field. There are only two possible values for $\left(v^{\nu}\right)^{2}: a_{0} b_{0}$ and $\left(a_{0}+n a_{1}\right)\left(b_{0}+n b_{1}\right)$. The first value corresponds to the case $\sum_{\beta} a_{\beta}^{\nu}=0$ and the second $\sum_{\beta} a_{\beta}^{\nu} \neq 0$. Hence, the first would be the spin velocity and the second the charge velocity. The Hamiltonian can now be written as

$$
H=\sum_{k}\left[\left(a_{0}+n a_{1}\right) \Pi_{k}^{c} \Pi_{-k}^{c}+\left(b_{0}+n b_{1}\right) k^{2} \phi_{k}^{c} \phi_{-k}^{c}+\sum_{s=1}^{n-1}\left\{a_{0} \Pi_{k}^{s} \Pi_{-k}^{s}+b_{0} k^{2} \phi_{k}^{s} \phi_{-k}^{s}\right\}\right]
$$

We have one charge mode and $n-1$ spin modes; and because of the $S U(n)$ symmetry all the spin modes have same velocity. We define the following velocities associated with $\Pi$ - and $\phi$-fields for the charge(denoted as c) and spin(denoted as s) modes: $v_{N}^{c}=a_{0}+n a_{1}, v_{J}^{c}=$ $b_{0}+n b_{1}, v_{N}^{s}=a_{0}, v_{J}^{s}=b_{0}$. The charge and spin velocities then are $v_{c}=\left(v_{N}^{c} v_{J}^{c}\right)^{1 / 2}$ and $v_{s}=\left(v_{N}^{s} v_{J}^{s}\right)^{1 / 2}$. 
Since the eigenstates for our model corresponds to the low energy excitations of the model, we can easily obtain the harmonic fluid parameters by examining the energy expanded about the ground state. By Taylor expansion we obtain the energy for the continuum model as follow,

$$
E=e_{0} N+\mu N+\frac{\pi \hbar \rho_{0}}{m} \frac{\pi \hbar}{L} \sum_{\sigma \sigma^{\prime}} \frac{1}{2} A_{\sigma \sigma^{\prime}} \Delta M_{\sigma} \Delta M_{\sigma^{\prime}}+2 B_{\sigma \sigma^{\prime}} \Delta J_{\sigma} \Delta J_{\sigma^{\prime}}
$$

where

$$
\begin{aligned}
e_{0} & =\frac{\left(\pi \hbar \rho_{0}\right)^{2}}{6 m}\left(\lambda+\frac{1}{n}\right)^{2}, \\
\mu & =\frac{\left(\pi \hbar \rho_{0}\right)^{2}}{2 m}\left(\lambda+\frac{1}{n}\right)^{2}, \\
A_{\sigma \sigma^{\prime}} & =\lambda\left(\lambda+\frac{1}{n}\right)+\left(\lambda+\frac{1}{n}\right) \delta_{\sigma \sigma^{\prime}}, \\
B_{\sigma \sigma^{\prime}} & =-\frac{\lambda}{n}+\left(\lambda+\frac{1}{n}\right) \delta_{\sigma \sigma^{\prime}},
\end{aligned}
$$

where $\rho_{0}=N / L$. We can now just read off the velocities,

$$
\begin{aligned}
& v_{s}=v_{N}^{s}=v_{J}^{s}=\frac{\pi \hbar \rho_{0}}{m}\left(\lambda+\frac{1}{n}\right) \\
& v_{N}^{c}=\frac{\pi \hbar \rho_{0}}{m} n\left(\lambda+\frac{1}{n}\right)^{2} \\
& v_{J}^{c}=\frac{\pi \hbar \rho_{0}}{m} \frac{1}{n} \\
& v_{c}=v_{s}
\end{aligned}
$$

The charge and spin velocities are same for all $\lambda$ and $n$. As expected from the singlet nature of the ground state the ratio $v_{J}^{s} / v_{N}^{s}$ does not get renormalized. The coefficient $v_{J}^{c}$ is independent of the interaction term, as a consequence of Galilean invariance, but $v_{N}^{c}$ gets renormalized due to non-linear interaction terms not included in the linear form of the harmonic Hamiltonian. The renormalization coupling constant for the charge is $\exp (-2 \varphi) \rightarrow\left(v_{N}^{c} / v_{J}^{c}\right)^{1 / 2}=n \lambda+1$ in the limit of long wavelength (i.e. $k \rightarrow 0$ ). Because of the scale invariance of the model the dimensionless coupling constant $\varphi$ is independent of the particle density.

The compressibility per particle is $\left(\pi^{2} \hbar^{2} \rho_{0}^{2} / m\right)(\lambda+1 / n)^{2}$. We also find the chemical potential and the ground state energy to be $m^{*} v_{F}^{2} / 2$ and $(N / 3)\left(m^{*} v_{F}^{2} / 2\right)$, respectively. The 
chemical potential (or the Fermi energy at zero temperature) and the ground state energy of this one-dimensional system is that of free fermions with the renormalized mass per particle $m^{*}=m /(n \lambda+1)^{2}$. The Fermi velocity is given by $v_{F}=\pi \hbar \rho_{0} /\left(n m^{*}\right)$.

The low energy excitation of the lattice version is given by Eq. (5.5) as well if we set $m=\hbar=1$ and replace $L, e_{0}$ and $\mu$ with $N_{a},-\pi^{2} / 3+2 e_{0}$, and $-\pi^{2} / 3+2 \mu$, respectively. The crucial difference is that this expression for the lattice model is true only for $\rho_{0}\left(=N / N_{a}\right) \leq$ $\rho_{0}^{\max }\left(=(l+1 / n)^{-1}\right)$. Hence, the charge velocity is linear in $\rho_{0}$ only up to $\rho_{0}^{\max }$. We expect $v_{c}$ to vanish as $\rho_{0} \rightarrow 1$ due to the metal-insulator transition at the density. $v_{c}$, therefore, should exhibit non-analyticity at $\rho_{0}^{\max }$. This behavior is attributed to the long-range interaction in our model. In ref. [4] this type of non-analyticity in the spinon velocity was observed for the $\mathrm{SU}(2)$ spin chain model. In the nearest neighbor interaction models the sharp change in the charge velocity is smoothed out.

For $l=1$ the energy for $\rho_{0} \geq(1+1 / n)^{-1}$ is explicitly found and the harmonic fluid Hamiltonian is given by Eq. (5.5) with $A_{\sigma \sigma^{\prime}}=\left(1-\rho_{0}\right)(n+2)-\rho_{0} / n+\delta_{\sigma \sigma^{\prime}}$ and $B_{\sigma \sigma^{\prime}}=$ $-\rho_{0} / n+\delta_{\sigma \sigma^{\prime}}$. Therefore, $v_{c}=\pi\left(1-\rho_{0}\right)(n-1)$ and $v_{s}=\pi$. The spin velocity is independent of the density in this region. Similarly, for $l=0$ we have $A_{\sigma \sigma^{\prime}}=B_{\sigma \sigma^{\prime}}=-\rho_{0} / n+\delta_{\sigma \sigma^{\prime}}$ for

$0 \leq \rho_{0} \leq 1$. Thus, $v_{c}=\pi\left(1-\rho_{0}\right)$ and $v_{s}=\pi$. This agrees with the results obtained by Kuramoto and Yokoyama [0]. However, it is unexpected that the charge and spin velocities are independent of the number of spin species. Fig. 4 shows the charge and spin velocities for the lattice models with various interaction parameters.

\section{CONCLUSION}

We have shown in this paper that the Sutherland's model of spinless particles interacting with inverse square exchange can be generalized to the multicomponent system of particles. We further generalized the continuum model to lattice for the integer values of the dimensionless interaction parameter. We explicitly constructed a class of eigenstates for these models and calculated the corresponding eigenenergies. We claimed in this article 
that the class of eigenstates correspond to the ground state and the low energy excitations of the model. We checked this by the exact numerical diagonalization of the lattice version of Hamiltonian for small systems. The systematic construction of the other eigenstates, thermodynamics, and the general n-point correlation functions for these models are yet to be found. As J. A. Minahan showed in his preprint [7] the Hamiltonian of the type given by Eq. (2.1) also arises in a matrix model as a representation of one dimensional open string theory.

This work is supported in part by NSF grant No. DMR-91-96212.

Note added: After the completion of this paper we received a preprint from N. Kawakami who reports similar results. And, the Hamiltonian given by Eq. (2.1) is also independently identified by A. P. Polychronakos [6].

\section{APPENDIX A: PROOF OF THEOREM 1}

We first discuss a diagrammatic way of writing the exchange operation. A labeled diagram is shown in Fig. 2. The amplitude of the labeled diagram shown in Fig. 20 can be evaluated using the following rules: i) the dashed line connecting the indices $i$ and $j$ gets a factor $z_{i} z_{j}\left(z_{i}-z_{j}\right)^{-2}\left(1-\delta_{\sigma, \sigma^{\prime}}\right) \delta_{\sigma \sigma_{i}} \delta_{\sigma \sigma_{j}}$, ii) each solid line connecting the indices $i$ and $\alpha_{k}$ gets a factor $\left(z_{i}-z_{j}\right)\left(z_{\alpha_{k}}-z_{i}\right)^{-1} \delta_{\sigma \sigma_{\alpha_{k}}}$, iii) each solid line connecting the indices $j$ and $\beta_{k}$ gets a factor $-\left(z_{i}-z_{j}\right)\left(z_{\beta_{k}}-z_{j}\right)^{-1} \delta_{\sigma \sigma_{\beta_{k}}}$. Weight of the dashed line vanishes whenever the spins at the sites $i$ and $j$ are same. On the other hand, weights of the solid lines that are connected to $i(j)$ vanish whenever the spins at the sites $\{\alpha\}(\{\beta\})$ and $i(j)$ are different. Hence, we may think of the diagram as two-spin interaction diagram. Since the Hamiltonian has only the two-spin exchange operator $P_{i j}$, three-spin and higher number of spin interactions are missing.

The amplitude of the labeled diagram is undesirable since it depends on the local variables. A more desirable, local variable independent diagram (unlabeled diagram) can be obtained by summing $(n+1)(m+1)$ cyclically permuted labeled diagrams. This fact will 
be proved in this appendix.

We note that the indices, $\left\{i, \alpha_{1}, \ldots, \alpha_{n}\right\}$ and $\left\{j, \beta_{1}, \ldots, \beta_{m}\right\}$, are dummy indices. Consequently, the sum will be invariant under any permutation of the indices. We further observe that $\Delta$ is invariant under any permutation of the indices except when there is interchange between the two sets, $\left\{i, \alpha_{1}, \ldots, \alpha_{n}\right\}$ and $\left\{j, \beta_{1}, \ldots, \beta_{m}\right\}$. On the other hand, the pre-factor to $\Delta$ remains invariant with respect to the permutations of indices $\{\alpha\}$ and $\{\beta\}$. It is convenient to consider only the cyclic permutations which give $(n+1)(m+1)$ distinct pre-factors. We, then, sum the $(n+1)(m+1)$ factors as follow:

$$
\begin{aligned}
P_{n m}^{\sigma \sigma^{\prime}} & =\sum_{i \neq j} \sum_{\{\alpha\},\{\beta\}} \frac{(-1)^{m}}{n ! m !} \frac{z_{i} z_{j}^{1-q}\left(z_{i}-z_{j}\right)^{n+m-2+q}}{\left(z_{i}-z_{\alpha_{1}}\right) \cdots\left(z_{i}-z_{\alpha_{n}}\right)\left(z_{j}-z_{\beta_{1}}\right) \cdots\left(z_{j}-z_{\beta_{m}}\right)} \Delta \\
& =\sum_{i \neq j\{\alpha\},\{\beta\}} \sum_{k, l} \frac{(-1)^{m}}{(n+1) !(m+1) !} \frac{z_{\gamma_{k}^{\alpha}} z_{\gamma_{l}^{\beta}}^{1-q}\left(z_{\gamma_{k}^{\alpha}}-z_{\gamma_{l}^{\beta}}\right)^{n+m-2+q}}{\left(z_{\gamma_{k}^{\alpha}}-z_{\alpha_{1}}\right) \cdots\left(z_{\gamma_{k}^{\alpha}}-z_{i}\right) \cdots\left(z_{\gamma_{k}^{\alpha}}-z_{\alpha_{n}}\right)} \\
& \times \frac{1}{\left(z_{\gamma_{l}^{\beta}}-z_{\beta_{1}}\right) \cdots\left(z_{\gamma_{l}^{\beta}}-z_{j}\right) \cdots\left(z_{\gamma_{l}^{\beta}}-z_{\beta_{m}}\right)} \Delta,
\end{aligned}
$$

where $\left\{\gamma^{\alpha}\right\} \equiv\left\{i, \alpha_{1}, \ldots, \alpha_{n}\right\}$ and $\left\{\gamma^{\beta}\right\} \equiv\left\{j, \beta_{1}, \ldots, \beta_{m}\right\}$.

We let $D_{\alpha}=\prod_{k<k^{\prime}}\left(z_{\gamma_{k}^{\alpha}}-z_{{k^{\prime}}^{\prime}}\right)$ and $D_{\beta}=\prod_{l<l^{\prime}}\left(z_{\gamma_{l}^{\beta}}-z_{\gamma_{l^{\prime}}^{\beta}}\right)$. Using the binomial theorem we expand the numerator in Eq. (A1). We then multiply and divide the expression by $D_{\alpha} D_{\beta}$ and obtain the following.

$$
P_{n m}^{\sigma \sigma^{\prime}}=\sum_{i \neq j} \sum_{\{\alpha\},\{\beta\}} \sum_{s=1}^{n+m-1+q} \frac{(-1)^{m-1+q-s}}{(n+1) !(m+1) !}\left(\begin{array}{c}
n+m-2+q \\
s-1
\end{array}\right) \frac{V_{\alpha}^{s} V_{\beta}^{n+m-s}}{D_{\alpha} D_{\beta}} \Delta,
$$

where

$$
V_{\alpha}^{s}=\left|\begin{array}{cccc}
1 & 1 & \ldots & 1 \\
z_{i} & z_{\alpha_{1}} & \ldots & z_{\alpha_{n}} \\
\vdots & \vdots & & \vdots \\
z_{i}^{n-1} & z_{\alpha_{1}}^{n-1} & \ldots & z_{\alpha_{n}}^{n-1} \\
z_{i}^{s} & z_{\alpha_{1}}^{s} & \ldots & z_{\alpha_{n}}^{s}
\end{array}\right|
$$

and $V_{\beta}^{k}$ is defined the same way. We further note that $V_{\alpha}^{s}=0$ if $0 \leq s \leq n-1$ and $V_{\beta}^{k}=0$ if $0 \leq k \leq m-1$. Therefore, if $0 \leq q \leq 1$, the only non-zero contribution in (A2) is the term with $s=n$. Since $V_{\alpha}^{n}=D_{\alpha}$ and $V_{\beta}^{m}=D_{\beta}$, we obtain 


$$
\begin{aligned}
P_{n m}^{\sigma \sigma^{\prime}} & =\frac{(-1)^{n+m-1+q}}{(n+1) !(m+1) !}\left(\begin{array}{c}
n+m-2+q \\
n-1
\end{array}\right) \sum_{i \neq j} \sum_{\{\alpha\},\{\beta\}} \Delta \\
& =(-1)^{n+m-1+q}\left(\begin{array}{c}
n+m-2+q \\
n-1
\end{array}\right)\left(\begin{array}{c}
M_{\sigma} \\
n+1
\end{array}\right)\left(\begin{array}{c}
M_{\sigma^{\prime}} \\
m+1
\end{array}\right) .
\end{aligned}
$$

Evaluating $\sum_{i \neq j} \sum_{\{\alpha\},\{\beta\}} \Delta$ in (A4) is same as calculating the total number of ways of putting $n+1$ out of $M_{\sigma}$ blue balls in a box and $m+1$ out of $M_{\sigma^{\prime}}$ red balls in another box. The sum, therefore, is equal to $M_{\sigma}\left(M_{\sigma}-1\right) \cdots\left(M_{\sigma}-n\right) M_{\sigma^{\prime}}\left(M_{\sigma^{\prime}}-1\right) \cdots\left(M_{\sigma^{\prime}}-m\right)$.

It is straightforward to prove the following useful identities:

$$
\begin{aligned}
\left(\begin{array}{c}
n+m-2+q \\
n-1
\end{array}\right) & =\sum_{s=0}\left(\begin{array}{c}
n-1 \\
s
\end{array}\right)\left(\begin{array}{c}
m-1+q \\
s
\end{array}\right), \\
(-1)^{s}\left(\begin{array}{c}
m-1+q \\
s
\end{array}\right) & =\sum_{k=0}^{s}(-1)^{k}\left(\begin{array}{c}
m+q \\
k
\end{array}\right), \\
& =\sum_{k=0}^{s}(-1)^{k}(s+1-k)\left(\begin{array}{c}
m+1+q \\
k
\end{array}\right), \\
\sum_{m=1-q}^{M_{\sigma}-1}(-1)^{m}\left(\begin{array}{c}
M_{\sigma} \\
m+1
\end{array}\right)\left(\begin{array}{c}
m+1 \\
k
\end{array}\right) & =\left\{\begin{array}{ccc}
-M_{\sigma}+1 & \text { if } \quad k=0 \\
-M_{\sigma} & \text { if } \quad k=1 \\
1 & \text { for } & k=0 \\
0 & \text { for } & 1 \leq k<M_{\sigma}
\end{array}\right\} \text { for } q=1,
\end{aligned}
$$

Using (A5) we may sum the terms depending on $n$ and $m$ in $P_{n m}^{\sigma \sigma^{\prime}}$ separately. Using (A6) and (A7) the following relation can easily be obtained:

$$
P^{\sigma \sigma^{\prime}}=\left\{\begin{array}{l}
-\sum_{k=1}^{\operatorname{Min}\left(M_{\sigma}, M_{\sigma^{\prime}}\right)}\left(M_{\sigma}-k\right)\left(M_{\sigma^{\prime}}-k\right) \text { for } q=0, \\
-\sum_{k=1}^{\operatorname{Min}\left(M_{\sigma}, M_{\sigma^{\prime}}\right)}\left(M_{\sigma}-k\right) \text { for } q=1 .
\end{array}\right.
$$

Q.E.D.

\section{APPENDIX B: PROOF OF THEOREM 2}

We replace the product over $k$ with two new sets of integer indices, $\{\alpha\}$ and $\{\beta\}$ and rewrite the expression in Theorem 2 as, 


$$
\begin{aligned}
Q & =\sum_{i \neq j} \sum_{\{\alpha\},\{\beta\}} \frac{1}{\left(M_{\sigma}-1\right) !\left(M_{\sigma^{\prime}}-1\right) !} \frac{z_{i}}{z_{j}}\left(\frac{z_{i}-z_{j}}{z_{j}}\right)^{q-2} \frac{z_{\alpha_{1}}-z_{j}}{z_{\alpha_{1}}-z_{i}} \frac{z_{\alpha_{2}}-z_{j}}{z_{\alpha_{2}}-z_{i}} \cdots \frac{z_{\alpha_{M_{\sigma}-1}}-z_{j}}{z_{\alpha_{M_{\sigma}-1}}-z_{i}} \\
& \times\left\{\sum_{r=0}^{M_{\sigma^{\prime}}-1}\left(z_{i}-z_{j}\right)^{r} f_{r}\left(\left\{z_{\beta}\right\}\right)\right\} \Delta .
\end{aligned}
$$

Here, $\Delta$ is same as in Theorem 1 . The factor in the curly bracket in (B1) is obtained by writing the terms like $\left(z_{\beta_{l}}-z_{i}\right) /\left(z_{\beta_{l}}-z_{j}\right)$ as $\left\{1-\left(z_{i}-z_{j}\right) /\left(z_{\beta_{l}}-z_{j}\right)\right\}$ and by multiplying out all the factors that depend on $\{\beta\} . f_{r}$ in $(\overline{\mathrm{B} 1})$ is some function of $\left\{z_{\beta}\right\}$, however, $f_{0}=1$.

As in Appendix A we rewrite the expression by summing over the factors obtained by the cyclic permutations of the indices $\left\{i, \alpha_{1}, \ldots, \alpha_{M_{\sigma}-1}\right\}$ and obtain

$$
Q=\sum_{i \neq j} \sum_{\{\alpha\},\{\beta\}} \sum_{r=0}^{M_{\sigma^{\prime}}-1} \frac{(-1)^{M_{\sigma}}}{M_{\sigma} !\left(M_{\sigma^{\prime}}-1\right) !}\left(z_{i}-z_{j}\right)\left(z_{\alpha_{1}}-z_{j}\right) \cdots\left(z_{\alpha_{M_{\sigma}}-1}-z_{j}\right) f_{r} \frac{W_{\alpha}^{M_{\sigma}}}{D_{\alpha}^{M_{\sigma}}},
$$

where $W_{\alpha}^{M_{\sigma}}$ is given by the Vandemonde determinant whose last row is modified to $z_{\gamma_{l}}\left(z_{\gamma_{l}}-\right.$ $\left.z_{j}\right)^{q+r-3} / z_{j}^{q-1},\left\{\gamma_{l}\right\} \equiv\left\{i, \alpha_{1}, \ldots, \alpha_{M_{\sigma}-1}\right\}$. It is straightforward to show that

$$
\begin{aligned}
W_{\alpha}^{M_{\sigma}} & =\left\{\begin{array}{l}
\frac{(-1)^{M_{\sigma}} D_{\alpha}^{M_{\sigma}}}{\left(z_{i}-z_{j}\right)\left(z_{\alpha_{1}}-z_{j}\right) \cdots\left(z_{\alpha_{M_{\sigma}}-1}-z_{j}\right)} \text { if } q=2 \text { and } r=0 \\
0 \text { if } 0 \leq q+r-3 \leq M_{\sigma}-3
\end{array}\right. \\
\sum_{i \neq j\{\alpha\},\{\beta\}} \sum_{0} \Delta & =M_{\sigma} ! M_{\sigma^{\prime}} !
\end{aligned}
$$

The only non-zero contribution to $Q$ is given by the condition $q=2$ and $r=0$. Since $0 \leq r \leq M_{\sigma^{\prime}}-1$, the sufficient condition for $W_{\alpha}^{M_{\sigma}}=0$ is $3 \leq q \leq M_{\sigma}-M_{\sigma^{\prime}}+1$. Therefore, we have for $M_{\sigma} \geq M_{\sigma^{\prime}}$

$$
Q=\left\{\begin{array}{l}
M_{\sigma^{\prime}} \text { for } q=2 \\
0 \text { for } 3 \leq q \leq M_{\sigma}-M_{\sigma^{\prime}}+1
\end{array}\right.
$$

Q.E.D. 


\section{REFERENCES}

[1] B. Sutherland, Phys. Rev. A 4, 2019 (1971); Phys. Rev. A 5, 1372 (1972).

[2] F. D. M. Haldane, Phys. Rev. Lett. 60, 635 (1988); B. Sriram Shastry, Phys. Rev. Lett. 60, 639 (1988).

[3] F. D. M. Haldane, Phys. Rev. Lett. 47, 1840 (1982); 48, 569(E) (1982).

[4] F. D. M. Haldane, Phys. Rev. Lett. 66, 1529 (1991).

[5] Y. Kuramoto and H. Yokoyama, Phys. Rev. Lett. 67, 1338 (1991).

[6] A. P. Polychronakos, preprint CU-TP-551.

[7] J. A. Minahan, preprint UVA-HET-92-01. 


\section{FIGURES}

FIG. 1. Evolution of the inverse square exchange models both in the continuum space and lattice. Models that already exist are in rectangular boxes and new models that are presented in this paper are in ovals. The models enclosed in the dotted box are continuum version and the rest of the models are lattice version. The solid arrows mean "evolved from" or "generalized from". The dashed arrows connect two equivalent models in disguise.

FIG. 2. Labeled diagram for $n \sigma$-spins and $m \sigma^{\prime}$-spins.

FIG. 3. Illustration of allowed values of the currents for the sector $\{14,12,10,6,4,2\}$. (a) Allowed. All currents are zero. (b) Allowed. Ground state for the sector. (c) Not allowed.

FIG. 4. Charge and spin velocities of the lattice models. (a) Fermionic supersymmetric t-J model. The velocities are independent of the number of spin species. (b) Bosonic supersymmetric t-J model. Up to the density $n /(1+n), v_{c}$ and $v_{s}$ are equal. (c) Bosonic and fermionic t-J models

with $l \geq 2$. The charge and spin velocities are same up to the density $(l+1 / n)^{-1}$. Beyond the density the ground state is no longer the Jastrow-product type. 
fig. 1 
Fig. 2

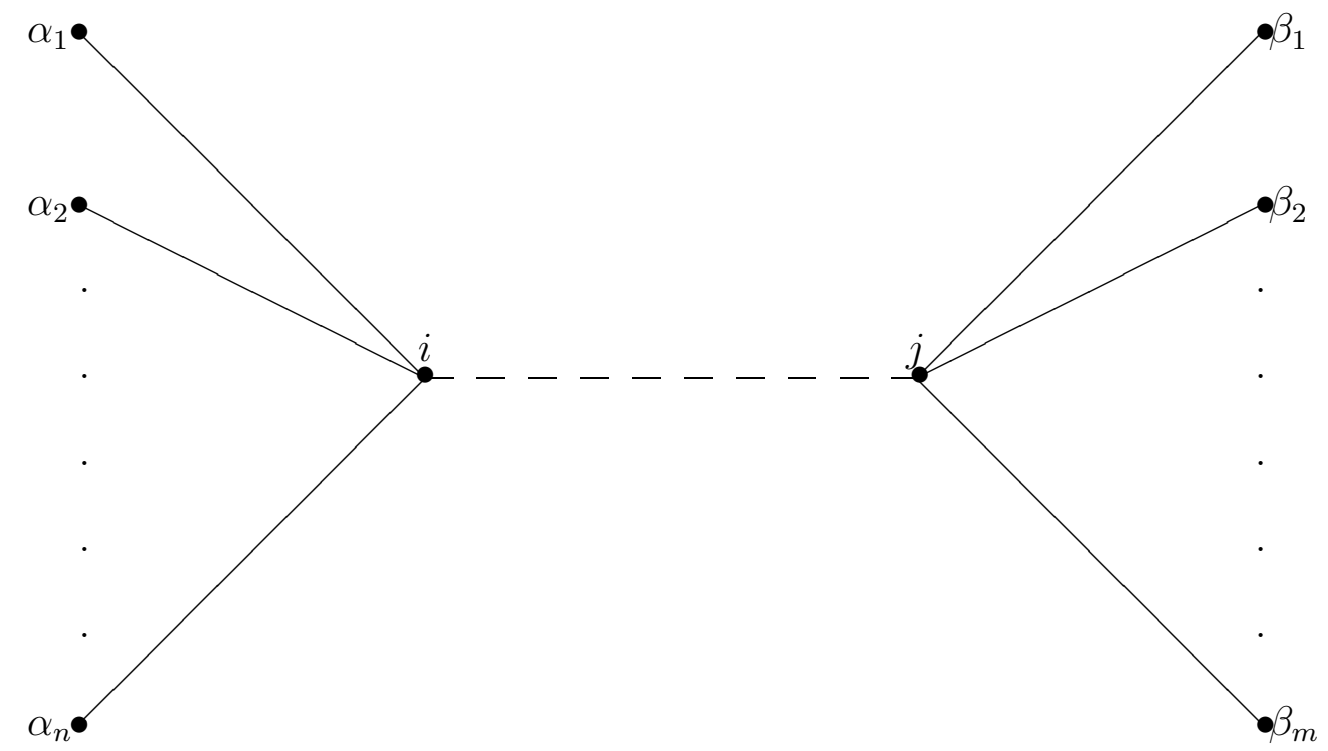


Fig. 3

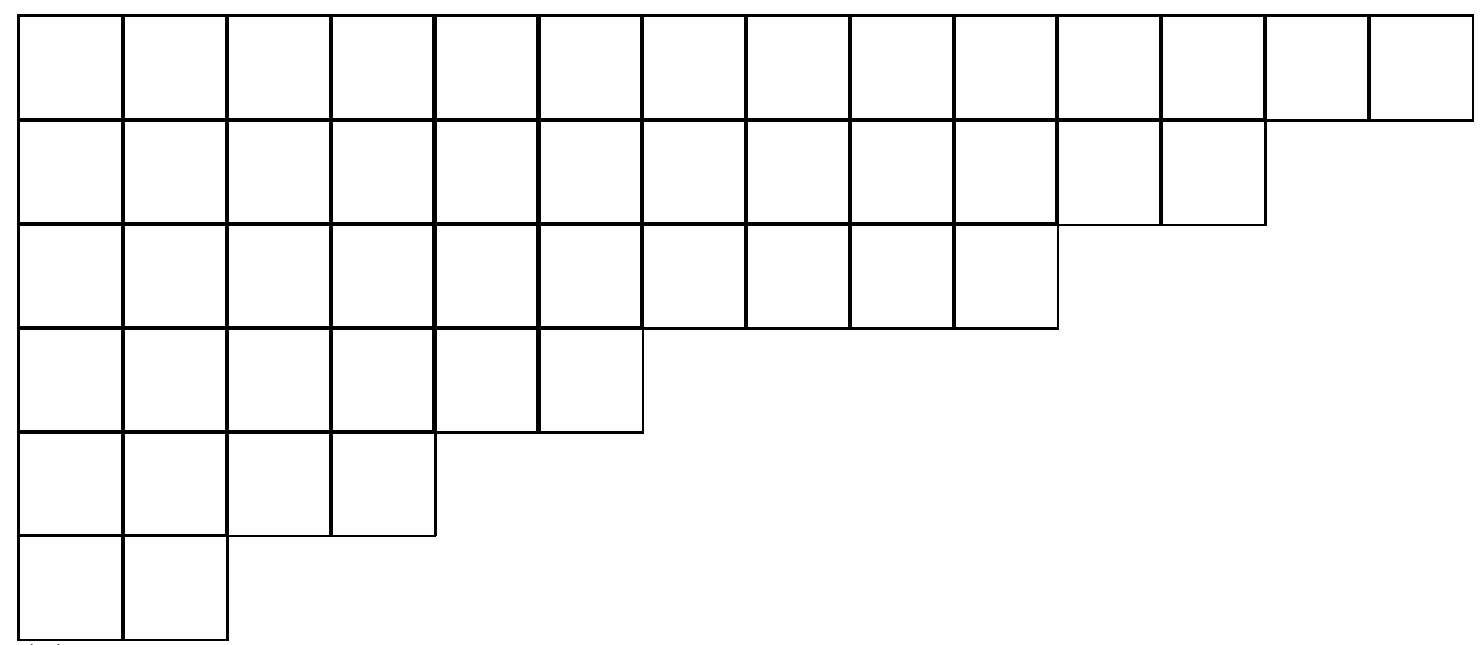

(a)

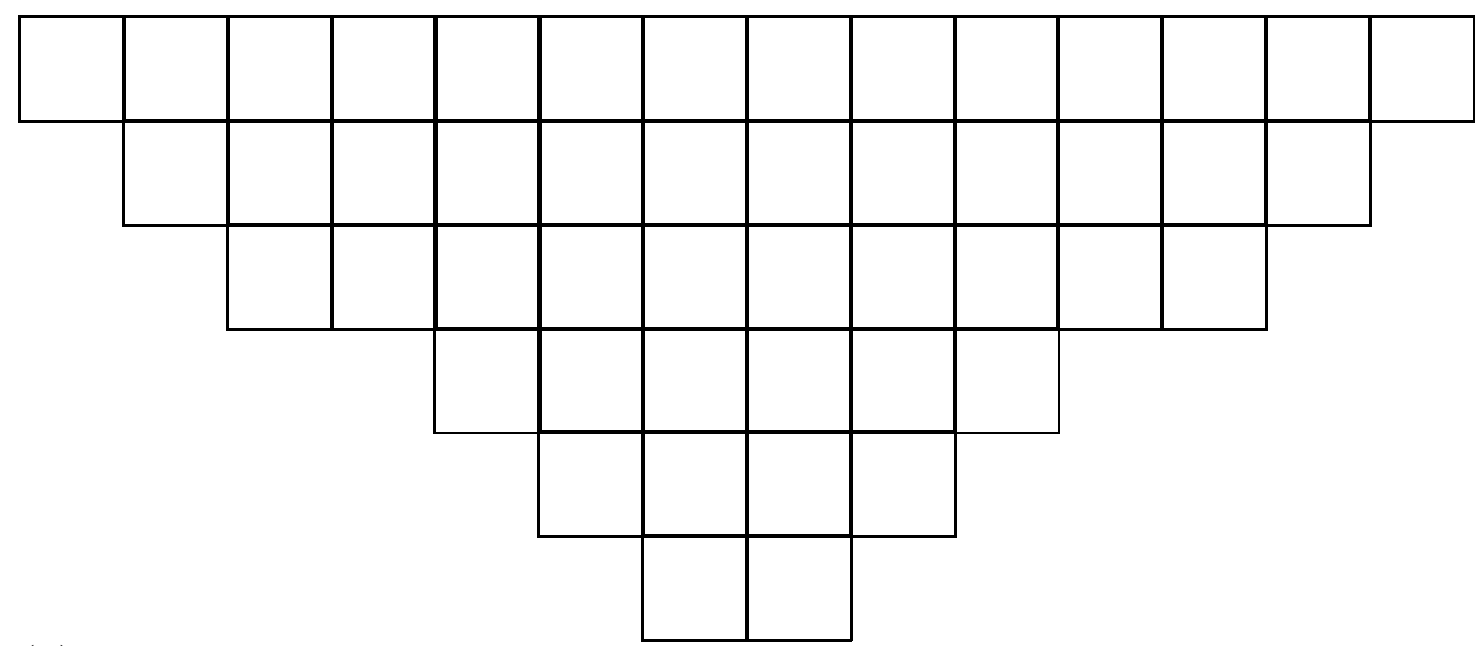

(b)

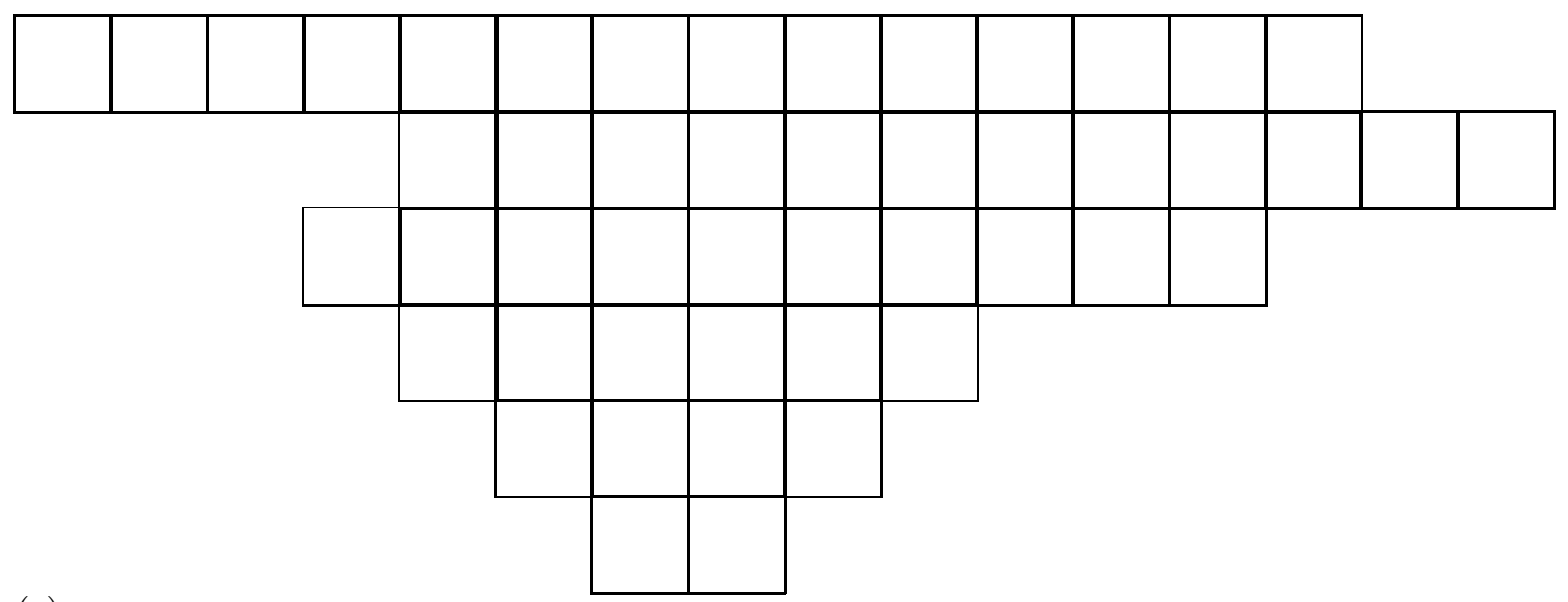

(c) 
fig. 4 